\section{DIN EN ISO 18153}

\section{G. Schumann}

Hannover, Deutschland

\section{Englischer Begriff ISO 18153}

Beschreibung Die internationale Norm „In-vitroDiagnostika - Messung von Größen in Proben biologischen Ursprungs - Metrologische Rückführbarkeit von Werten der katalytischen Konzentration von Enzymen, die Kalibratoren und Kontrollmaterialien zugeordnet sind" enthält die speziellen Anforderungen für die metrologische Rückführung der Messwerte für katalytische Enzymkonzentrationen. Die Messgröße „Katalytische Enzymkonzentration“ wird durch die sehr detaillierte Beschreibung der Messbedingungen defi- niert. Damit unterscheidet sich diese Messgröße von methodenunabhängigen Messgrößen, deren Bedingungen für die metrologische Rückführung in ISO 17511 ( $\triangleright$ DIN EN ISO/IEC 17511) beschrieben sind.

Die Inhalte dieser Norm werden in die revidierte Version der DIN EN ISO 17511 eingefügt. Nach der Verabschiedung der revidierten Norm DIN EN ISO 17511 wird die Bearbeitung von DIN EN ISO 18153 eingestellt.

\section{Literatur}

DIN EN ISO 18153 (2003) In-vitro-Diagnostika - Messung von Größen in Proben biologischen Ursprungs - Metrologische Rückführbarkeit von Werten der katalytischen Konzentration von Enzymen, die Kalibratoren und Kontrollmaterialien zugeordnet sind. Beuth-Verlag, Berlin 